\title{
A Pedagogical Approach to Obtain the Combined First and Second Law of Thermodynamics from Classical Statistical Mechanics
}

\author{
Ananth Govind Rajan* \\ Department of Chemical Engineering, Indian Institute of Science, Bengaluru, Karnataka \\ 560012, India \\ E-mail: ananthgr@iisc.ac.in
}

\begin{abstract}
The combined first and second law of thermodynamics for a closed system is written as $d E=T d S-P d V$, where $E$ is the internal energy, $S$ is the entropy, $V$ is the volume, $T$ is the temperature, and $P$ is the pressure of the system. This equation forms the basis for understanding physical phenomena ranging from heat engines to chemical reactors to biological systems. In this work, we present a pedagogical approach to obtain the combined first and second law of thermodynamics beginning with the principles of classical statistical mechanics, thereby establishing a fundamental link between energy conservation, heat, work, and entropy. We start with Boltzmann's entropy formula and use differential calculus to establish this link. Some new aspects of this work include the use of the microcanonical ensemble, which is typically considered to be intractable, to write the partition function for a general system of matter; deriving the average of the inverse kinetic energy, which appears in the microcanonical formulation of the combined first and second law, and showing that it is equal to the inverse of the average kinetic energy; obtaining an expression for the pressure of a system involving many-body
\end{abstract}


interactions; and introducing the system pressure in the combined first and second law via Clausius's virial theorem. Overall, this work informs the derivation of fundamental thermodynamic relations from an understanding of classical statistical mechanics. The material presented herein could be incorporated into senior undergraduate/graduatelevel courses in statistical thermodynamics and/or molecular simulations.

\section{Introduction}

Thermodynamics and statistical mechanics are important branches of science that enable the description of physical, $\frac{122}{2}$ chemical, $\frac{3,5}{5}$ and biological $\frac{617}{6}$ phenomena occurring around us. Moreover, statistical mechanics forms the basis for molecular and atomic-scale simulations of physicochemical systems using computers. $\frac{819}{}$ Thermodynamics has even pervaded fields such as information theory ${ }^{10}$ and networks. ${ }^{11}$ Therefore, the teaching and learning of thermodynamics and statistical mechanics form an important component of the senior undergraduate and graduate curriculum in chemistry and chemical engineering. The basis for thermodynamics are three laws dealing with quantities such as internal energy, entropy, heat, and work that have been formulated and refined over the course of many centuries. $\frac{12}{12}$ The first law is, simply stated, the conservation of energy, but when written in a differential form and combined with the second law, it conveys more information than that by introducing concepts such as temperature, entropy, pressure, and volume in the description of energy conservation.

The development of statistical mechanics by Gibbs, Boltzmann, Maxwell, Planck, and many other researchers led to a link between the microscopic realm of atoms and molecules, and the macroscopic realm of temperature, pressure, and volume. The three original statisticalmechanical "ensembles" introduced by Gibbs - canonical, microcanonical, and grand-canonicalare still used today to understand thermodynamic systems. $\stackrel{13}{13}$ Over the years, there has been much interest in rationalizing and teaching the laws of thermodynamics via an understanding of statistical mechanics. ${ }^{14}$ Such an understanding would help improve learning outcomes for 
students by presenting an innate link between the microscopic and macroscopic realm. In terms of previous research in this area, Lieb and Yngvason discussed the mathematical and physical foundations of the second law of thermodynamics. ${ }^{15}$ Later, Gemmer et al. derived a version of the second law of thermodynamics for quantum systems, $\frac{16}{16}$ an aspect which was also explored by Tasaki some years ago. $\frac{17}{17}$ In other work, Masanes and Oppenheim provided a derivation of the third law of thermodynamics using the principles of quantum and statistical mechanics. $\frac{18}{18}$ Very recently, Strasberg and Winter derived the first and second law of thermodynamics for quantum systems. $\frac{19}{19}$ With respect to the thermodynamics of classical fluids, Swaney and Bird obtained the first and second laws starting from the continuity and momentum conservation equations. .20 Nevertheless, a derivation of the combined first and second law of classical thermodynamics, using the basic principles of statistical mechanics is lacking, although authors have shown that statistical mechanics is consistent with the former, via the canonical ensemble. ${ }^{21 / 22}$ Such a derivation would be particularly useful in the teaching and learning of statistical thermodynamics.

To this end, in this work, we present a pedagogical approach to obtain the combined first and second law of thermodynamics starting from the microcanonical partition function of matter. Although some parts of the derivation presented here may be found in the widely used books of the field, $\frac{1321,23}{23}$ some new aspects of this work are:

- Use of the microcanonical ensemble, which is typically considered to be intractable, to write the partition function for a general system of matter.

- Deriving the average of the inverse kinetic energy, which appears in the microcanonical formulation of the combined first and second law, and showing that it is equal to the inverse of the average kinetic energy.

- Obtaining an expression for the pressure of a system involving many-body interactions.

- Introducing the system pressure in the combined first and second law via Clausius's virial theorem. 
We hope that the new understanding synthesized here can aid the use of the microcanonical ensemble to derive thermodynamic and statistical-mechanical relations, and elucidate the link between statistical mechanics and the combined first and second law of thermodynamics.

\section{The combined first and second law of thermodynamics}

The first law of thermodynamics is essentially a statement of energy conservation, and establishes the equivalence between work and heat in terms of bringing about energy changes in a specified system. In mathematical terms, the first law for a closed system (i.e., one that cannot exchange mass) is written as:

$$
d E=\delta Q+\delta W
$$

where $E$ is the internal energy of the system and $\delta Q$ and $\delta W$ represent, respectively, the heat added to and work done on, the system. The symbol $\delta$ is used to indicate infinitesimal quantities of heat and work, instead of $d$, because heat and work are not necessarily state functions, i.e., they depend on the path taken by the system during a thermodynamic trans-

formation. The second law of thermodynamics states that for any process, $d S \geq \frac{\delta Q}{T}$, where $S$ is the entropy and $T$ is the temperature of the system, and the equality only holds for reversible, i.e., quasi-static processes. For reversible processes, one can thus write $\delta Q=T d S$ and $\delta W=-P d V$, so that:

$$
d E=T d S-P d V
$$

where $V$ is the volume and $P$ is the pressure of the system. This expression is called the combined first and second law of thermodynamics. Although written for a reversible process, the above equation is valid for any process, because $E, T, S, P$, and $V$ are state functions. In this work, our objective is to establish the above mathematical expression using the principles of statistical mechanics. 


\section{The microcanonical ensemble}

Since the combined first and second law for a closed system involves the differentials $d E$, $d S$, and $d V$ at constant $N$, the microcanonical $(N V E)$ ensemble involving fixed $N, V$, and $E$ is well suited for use in the current work. However, historically, the microcanonical ensemble has been avoided because it is mathematically inconvenient as compared to the canonical ensemble (i.e., when the system is at constant $N V T) \cdot{ }^{22}$ In this work, apart from concepts from classical mechanics, such as, the kinetic energy, potential energy, and the system Hamiltonian, we start with two other postulates:

- The Boltzmann entropy formula

- The principle of entropy maximization

According to the Boltzmann entropy formula, $\underline{24}$ the entropy $S$ of a system is given as

$$
S=k_{B} \ln \Omega
$$

where $k_{B}$ is the Boltzmann constant and $\Omega$, which is referred to as the "partition function", is the number of "microstates" in the "phase space" formed by the momenta $\left(\mathbf{p}^{N}\right)$ and positions $\left(\mathbf{r}^{N}\right)$ of the particles, corresponding to a fixed value of $N, V$, and $E$. Note that Swendsen argued that it is more appropriate for $\Omega$ to represent the area of a constant energy surface, thereby resulting in the Boltzmann surface entropy, $\stackrel{24}{2}$ rather than the volume of a constant energy surface, resulting in Gibbs's volume entropy. ${ }^{25}$ Accordingly, we use the former definition of the partition function in this work, an argument also supported by Frenkel and Warren. ${ }^{26}$ Further, the principle of entropy maximization states that absent any internal restraints on a system, the system always tends to the state of maximum entropy. Here, we show that these two postulates, along with the principles of classical mechanics, can be used to obtain the combined first and second law of thermodynamics. 


\section{The canonical ensemble and the concept of temperature}

To define the concept of temperature, we consider a composite, isolated system made of the system of interest $(I)$ and a large thermal reservoir $(R)$, as represented schematically in Figure 1. We adapt the discussion outlined in Pathria and Beale, $\underset{27}{[27}$ Reif,$\frac{28}{28}$ and other books. Although the link between temperature and statistical mechanics is well known, it is instructive to reiterate it, to understand that the Boltzmann entropy formula, along with the principle of entropy maximization, contains all information required to derive the combined first and second law of thermodynamics.

The energies of the system of interest and the reservoir are denoted as $E_{I}$ and $E_{R}$, respectively. Similarly, the number of their microstates are denoted as $\Omega_{I}$ and $\Omega_{R}$. When any internal restraints (e.g., those preventing the free transfer of energy) are removed, the composite system will tend to a state of maximum entropy, subject to the constraint $E_{I}+$ $E_{R}=E$, where $E$, the total energy of the composite system is a constant, as the latter is isolated. Since the number of microstates are multiplicative, $\Omega=\Omega_{I} \Omega_{R}$. It follows that the entropy, $S$, of the composite system is given as:

$$
S=k_{B} \ln \left(\Omega_{I} \Omega_{R}\right)=k_{B}\left(\ln \Omega_{I}+\ln \Omega_{R}\right) .
$$

We will work with dimensionless quantities. Thus, we consider:

$$
\frac{S}{k_{B}}=\ln \Omega_{I}+\ln \Omega_{R} .
$$

Now, at equilibrium, in the absence of any internal restraints, the entropy of the composite system will tend to its maximum allowable value. Thus, we need to maximize $S / k_{B}$ subject to the constraint $E_{I}+E_{R}=E$. Using the method of Lagrange multipliers, we can define the Lagrangian as:

$$
\mathcal{L}=\ln \Omega_{I}+\ln \Omega_{R}+\beta\left(E-E_{I}-E_{R}\right),
$$




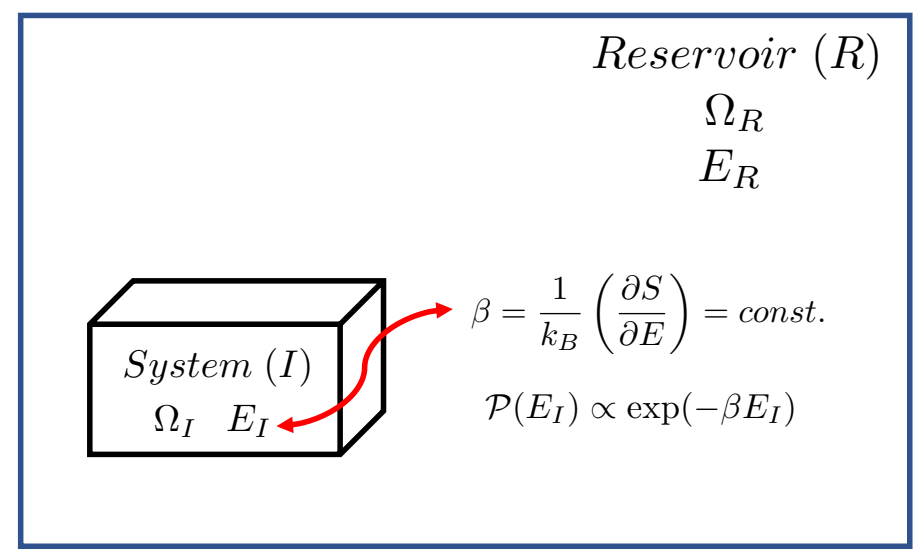

Figure 1: The concept of temperature in statistical mechanics. The system of interest $(I)$ and a large thermal reservoir $(R)$ form a composite system that is isolated (indicated by a purple border). The number of microstates of the two are denoted by $\Omega_{I}$ and $\Omega_{R}$ and their energies by $E_{I}$ and $E_{R}$, respectively. At equilibrium, $\beta=\frac{1}{k_{B} T}$ equalizes after exchange of energy (indicated by the red arrow) and the probability of the system of interest having energy $E_{I}$ is proportional to the Boltzmann factor $\exp \left(-\beta E_{I}\right)$.

where $\beta$ is a Lagrange multiplier with units of inverse energy for the equation to be dimensionally consistent. For satisfying the maximum condition, we can write:

$$
\begin{aligned}
& \frac{\partial \mathcal{L}}{\partial E_{I}}=0 \Rightarrow \frac{\partial \ln \Omega_{I}}{\partial E_{I}}-\beta=0 \\
& \frac{\partial \mathcal{L}}{\partial E_{R}}=0 \Rightarrow \frac{\partial \ln \Omega_{R}}{\partial E_{R}}-\beta=0 .
\end{aligned}
$$

Thus, at equilibrium (see Figure 1), we have:

$$
\beta=\frac{\partial \ln \Omega}{\partial E}=\frac{1}{k_{B}} \frac{\partial S}{\partial E}=\text { constant. }
$$

Since, empirically, the temperature of two systems equalize at equilibrium, we infer that $\beta$ is a quantity related to the temperature of the system. Although we have obtained this relation via entropy maximization, we find later that this relation also falls out of the microcanonical partition function for any kind of matter composed of atoms (see Eq. (50p). 
Next, we consider the probability, $\mathcal{P}\left(E_{I}\right)$, that the system of interest has energy $E_{I}$ :

$$
\mathcal{P}\left(E_{I}\right)=C \Omega_{I}\left(E_{I}\right)
$$

where $C$ is a normalization constant. The number of microstates with the system of interest having energy $E_{I}$ is the same as those with the reservoir having energy $\left(E-E_{I}\right)$. Thus,

$$
\mathcal{P}\left(E_{I}\right)=C \Omega_{R}\left(E-E_{I}\right) .
$$

Taking the logarithm on both sides, we have:

$$
\ln \mathcal{P}\left(E_{I}\right)=\ln C+\ln \Omega_{R}\left(E-E_{I}\right) .
$$

Since the thermal reservoir, i.e., heat bath, is much larger than the system of interest, $E_{I}<<E$, whence we can use a Taylor expansion to first order to get:

$$
\ln \mathcal{P}\left(E_{I}\right)=\ln C+\left[\ln \Omega_{R}(E)-E_{I} \frac{\partial \ln \Omega_{R}}{\partial E_{R}}\right] .
$$

Recognizing that $\beta=\frac{\partial \ln \Omega_{R}}{\partial E_{R}}$ and exponentiating both sides, we obtain:

$$
\mathcal{P}\left(E_{I}\right)=C \Omega_{R}(E) \exp \left(-\beta E_{I}\right)
$$

Thus,

$$
\mathcal{P}\left(E_{I}\right) \propto \exp \left(-\beta E_{I}\right)
$$

which is the defining equation for the Boltzmann distribution that is followed by systems in the canonical ensemble (see Figure 1). We note that the Boltzmann distribution can also be obtained via a combinatorial approach, by maximizing the number of ways of arranging the particles among the system's available energy levels given a fixed amount of energy, as 
illustrated by Tolman $\underline{29}$ and Hill. $\stackrel{22}{22}$

Finally, the link between $\beta$ and temperature can be made via the ideal gas law $(P V=$ $\left.N k_{B} T\right)$, which can be derived using statistical mechanics to be $P V=\frac{N}{\beta}$, so that $\beta=\frac{1}{k_{B} T}$. $\frac{29130}{}$

\section{The partition function in the microcanonical ensemble}

We consider a monoatomic system of $N$ atoms having equal mass inside a cuboidal box of dimensions $L_{x} \times L_{y} \times L_{z}$ (see Figure 2). The Hamiltonian of this system can be expressed as:

$$
\mathcal{H}=\sum_{i=1}^{N} \frac{\mathbf{p}_{i}^{2}}{2 m}+U\left(\mathbf{r}^{N}\right)
$$

where $\mathbf{p}_{i}$ denotes the momentum of atom $i, m$ denotes the mass of each particle, and $U$ denotes the potential energy of the system, which is a function of the atomic coordinates, $\mathbf{r}^{N}$. Note that, if required, the assumption of equal atomic masses may be relaxed by redefining $\frac{\mathbf{p}}{\sqrt{m_{i}}}\left(\right.$ where $m_{i}$ is the mass of atom $i$ ) to be the variable of integration over the phase space in Eq. (17) below.

In classical statistical mechanics, $\Omega$ is calculated as an integral over the phase space as

$$
\Omega=\frac{1}{h^{3 N} N !} \int d \mathbf{p}^{N} d \mathbf{r}^{N} \delta(E-\mathcal{H}) \Delta E
$$

where $h$ is the Planck constant (the factor $h^{3 N}$ was introduced a posteriori to account for the discretization of the phase space), $N$ ! accounts for the particles (atoms in our case) being indistinguishable, $\delta$ is the Dirac delta function, and $\Delta E$ is the allowable spread in the energy, $E$, in the microcanonical ensemble, which is taken to be infinitesimally small.

Using $\mathcal{H}$ from Eq. (16), we have

$$
\Omega=\frac{1}{h^{3 N} N !} \int d \mathbf{p}^{N} d \mathbf{r}^{N} \delta\left(E-\sum_{i=1}^{N} \frac{\mathbf{p}_{i}^{2}}{2 m}-U\right) \Delta E
$$




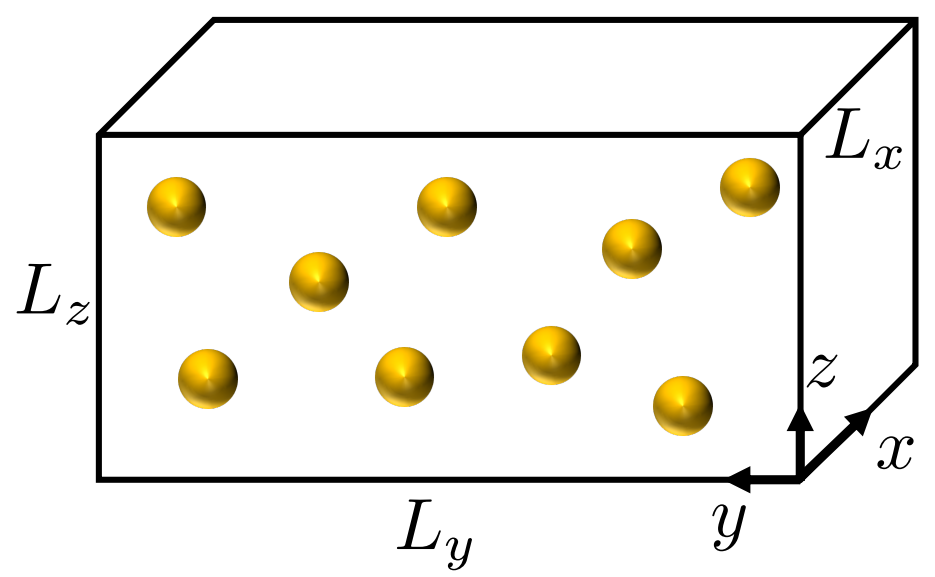

Figure 2: The system of interest. We consider matter consisting of monoatomic species of mass $m$ present inside a cuboidal system of dimensions $L_{x} \times L_{y} \times L_{z}$. The number of particles is denoted by $N$ and the volume of the system by $V$. The collisions of the particles result in a pressure $P$ in the bulk and at the boundary of the system.

where $U \equiv U\left(\mathbf{r}^{N}\right)$. It follows that:

$$
\Omega=\frac{1}{h^{3 N} N !} \int d \mathbf{p}^{N} d \mathbf{r}^{N} \delta\left(\frac{2 m(E-U)-\sum_{i=1}^{N} \mathbf{p}_{i}^{2}}{2 m}\right) \Delta E
$$

Using the properties $\delta\left(\frac{x}{a}\right)=|a| \delta(x)$ and $\delta(-x)=\delta(x)$, we obtain:

$$
\Omega=\frac{1}{h^{3 N} N !} \int d \mathbf{p}^{N} d \mathbf{r}^{N} \delta\left(\sum_{i=1}^{N} \mathbf{p}_{i}^{2}-2 m(E-U)\right) 2 m \Delta E
$$

Note that the constant of motion

$$
\sum_{i=1}^{N} \mathbf{p}_{i}^{2}-\sqrt{2 m(E-U)}^{2}=0
$$

represents the equation of a "hypersphere" of radius $r_{E}=\sqrt{2 m(E-U)}$ in $3 N$ dimensional space. Note that a hypersphere is the extension of the concept of a sphere from three dimensions to $3 \mathrm{~N}$ dimensions. Thus, $\int d \mathbf{p}^{N} \delta\left(\sum_{i=1}^{N} \mathbf{p}_{i}^{2}-\sqrt{2 m(E-U)}^{2}\right) 2 m \Delta E$ represents 


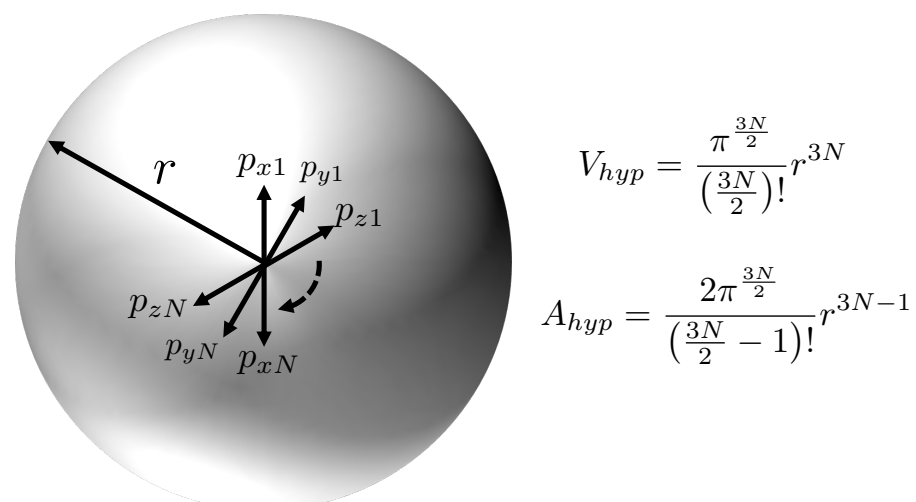

Figure 3: The concept of the momentum hypersphere. The momenta of the particles in the three Cartesian directions $\left(p_{x 1}, p_{y 1}, p_{z 1}, p_{x 2}, p_{y 2}, p_{z 2}, \ldots, p_{x N}, p_{y N}, p_{z N}\right)$ form a $3 N$ dimensional hypersphere of radius $r$ in momentum space. The expressions for the volume $\left(V_{h y p}\right)$ and the surface area $\left(A_{h y p}=\frac{d V_{h y p}}{d r}\right)$ of the hypersphere in terms of the radius are indicated. One may check that these equations reduce to the well-known expressions $V_{\text {sphere }}=$ $\frac{4}{3} \pi r^{3}$ and $A_{\text {sphere }}=4 \pi r^{2}$ for spheres. The area of the hypersphere is used in determining the microcanonical partition function and the average inverse kinetic energy of the system.

a volume element of that hypersphere (see Figure 3), and is given as:

$$
A_{\text {hyp }} d p=\frac{2 \pi^{\frac{3 N}{2}}}{\left(\frac{3 N}{2}-1\right) !} r_{E}^{3 N-1} d p
$$

where $p^{2}=\sum_{i=1}^{N} \mathbf{p}_{i}^{2}=2 m(E-U)$, so that $d p=\frac{2 m \Delta E}{2 \sqrt{2 m(E-U)}}$.

It follows that:

$$
\Omega=\frac{1}{h^{3 N} N !} \int d \mathbf{r}^{N} \frac{(2 \pi m)^{\frac{3 N}{2}}}{\left(\frac{3 N}{2}-1\right) !}(E-U)^{\frac{3 N}{2}-1} \Delta E
$$

which is dimensionally consistent. Defining

$$
\kappa=\frac{1}{h^{3 N} N !} \frac{(2 \pi m)^{\frac{3 N}{2}}}{\left(\frac{3 N}{2}-1\right) !} \Delta E
$$


we have

$$
\Omega=\kappa \int d \mathbf{r}^{N}(E-U)^{\frac{3 N}{2}-1}
$$

We now convert the positional coordinates of the particles, $\mathbf{r}_{i}$, to their fractional forms, $\mathbf{s}_{i}$ (see, e.g., McQuarrie ${ }^{21}$ ). This step is essential to be able to differentiate the partition function with respect to the volume of the system. To this end, we proceed by normalizing the Cartesian coordinates of the particles $\left(r_{x i}, r_{y i}, r_{z i}\right)$ with the respective dimension of the system $\left(L_{x}\right.$ or $L_{y}$ or $\left.L_{z}\right)$, such that: $s_{x i}=\frac{r_{x i}}{L_{x}}, s_{y i}=\frac{r_{y i}}{L_{y}}$, and $s_{z i}=\frac{r_{z i}}{L_{z}}$. The partition function thus reduces to

$$
\Omega=\kappa \int d \mathbf{s}^{N}\left(L_{x} L_{y} L_{z}\right)^{N}(E-U)^{\frac{3 N}{2}-1}
$$

Recognizing that $L_{x} L_{y} L_{z}$ is the volume of the system, $V$, we have:

$$
\Omega=\kappa V^{N} \int d \mathbf{s}^{N}(E-U)^{\frac{3 N}{2}-1}
$$

The physical meaning of this equation can be understood in terms of both interparticle interactions and external forces. For fixed $N, V$, and $E, \Omega$ is highest when $U<0$, followed by when $U=0$, and is the least when $U>0$. This makes physical sense, because attractive interactions between particles $(U<0)$ increase entropy by promoting mixing while repulsive interactions $(U>0)$ reduce entropy by seeking to prevent the particles from mixing. Similarly, external forces that stablize the system $(U<0)$ lead to an increase in entropy by increasing the kinetic energy of the particles for fixed $E$. Put another way, any destabilizing interactions or external forces (leading to $U>0$ ) seek to act as a constraint, and reduce the entropy of the system from its maximum value. Thus, we have shown the postulate of entropy maximization to naturally follow from the Boltzmann entropy formula and the principles of classical mechanics. One can write the above equation as:

$$
\Omega=\int d \mathbf{s}^{N} \Omega^{\prime}\left(\mathbf{s}^{N}\right)
$$


where $\Omega^{\prime}=\kappa V^{N}(E-U)^{\frac{3 N}{2}-1}$. It follows that the weighted average of any quantity $A$ over the phase space can be defined as:

$$
\langle A\rangle=\frac{\int d \mathbf{s}^{N} A\left(\mathbf{s}^{N}\right) \Omega^{\prime}\left(\mathbf{s}^{N}\right)}{\int d \mathbf{s}^{N} \Omega^{\prime}\left(\mathbf{s}^{N}\right)}=\frac{\int d \mathbf{s}^{N} A\left(\mathbf{s}^{N}\right) \Omega^{\prime}\left(\mathbf{s}^{N}\right)}{\Omega} .
$$

\section{The internal energy differential}

To write the first law of thermodynamics in differential form, we turn to differential calculus. Given that one needs two variables to characterize the state of a single-component, single-phase, closed system (because $N$ is fixed), and that we have chosen to work in the microcanonical ensemble, we have that

$$
E \equiv E(S, V)
$$

Thus, we can write

$$
d E=\left(\frac{\partial E}{\partial S}\right)_{V} d S+\left(\frac{\partial E}{\partial V}\right)_{S} d V
$$

To establish Eq. (2), we need to evaluate the partial derivatives $\left(\frac{\partial E}{\partial S}\right)_{V}$ and $\left(\frac{\partial E}{\partial V}\right)_{S}$. However, since the microcanonical ensemble only directly affords the calculation of entropy, we cast these partial derivatives in a form that is amenable to evaluate using the Boltzmann entropy formula, i.e., $S=k_{B} \ln \Omega$. Firstly, one can write

$$
\left(\frac{\partial E}{\partial S}\right)_{V}=\frac{1}{\left(\frac{\partial S}{\partial E}\right)_{V}}
$$

Secondly, using the triple product rule of partial derivatives, we have

$$
\left(\frac{\partial E}{\partial V}\right)_{S}=\frac{-1}{\left(\frac{\partial V}{\partial S}\right)_{E}\left(\frac{\partial S}{\partial E}\right)_{V}}=\frac{-\left(\frac{\partial S}{\partial V}\right)_{E}}{\left(\frac{\partial S}{\partial E}\right)_{V}}
$$


Thus, to obtain the total internal energy differential $d E$, one needs to evaluate the partial derivatives $\left(\frac{\partial S}{\partial E}\right)_{V}$ and $\left(\frac{\partial S}{\partial V}\right)_{E}$. Firstly

$$
\left(\frac{\partial S}{\partial E}\right)_{V}=\left[\frac{\partial}{\partial E}\left(k_{B} \ln \Omega\right)\right]_{V}
$$

or,

$$
\left(\frac{\partial S}{\partial E}\right)_{V}=\frac{k_{B}}{\Omega}\left(\frac{\partial \Omega}{\partial E}\right)_{V}
$$

Using $\Omega$ from Eq. (27), we have:

$$
\left(\frac{\partial S}{\partial E}\right)_{V}=\frac{k_{B}}{\Omega}\left[\frac{\partial}{\partial E}\left(\kappa V^{N} \int d \mathbf{s}^{N}(E-U)^{\frac{3 N}{2}-1}\right)\right]_{V} .
$$

Moving the partial derivative inside the integral, it follows that

$$
\left(\frac{\partial S}{\partial E}\right)_{V}=\left(\frac{3 N}{2}-1\right) k_{B} \frac{\int d \mathbf{s}^{N} \frac{1}{E-U} \kappa V^{N}(E-U)^{\frac{3 N}{2}-1}}{\Omega} .
$$

Note that $K=E-U$. Further, as per Eq. (29), the fraction that appears in the above equation defines the average of $\frac{1}{E-U}$ over the phase space, so that:

$$
\left(\frac{\partial S}{\partial E}\right)_{V}=\left(\frac{3 N}{2}-1\right) k_{B}\left\langle\frac{1}{K}\right\rangle
$$

which for large $N$ becomes:

$$
\left(\frac{\partial S}{\partial E}\right)_{V}=\frac{3 N k_{B}}{2}\left\langle\frac{1}{K}\right\rangle
$$

Next, we evaluate $\left(\frac{\partial S}{\partial V}\right)_{E}$ as follows:

$$
\left(\frac{\partial S}{\partial V}\right)_{E}=\frac{k_{B}}{\Omega}\left(\frac{\partial \Omega}{\partial V}\right)_{E}
$$


Again, using $\Omega$ from Eq. (27), we have:

$$
\left(\frac{\partial S}{\partial V}\right)_{E}=\frac{k_{B}}{\Omega}\left[\frac{\partial}{\partial V}\left(\kappa V^{N} \int d \mathbf{s}^{N}(E-U)^{\frac{3 N}{2}-1}\right)\right]_{E}
$$

We now use the product rule for differentiation to obtain

$$
\begin{array}{r}
\left(\frac{\partial S}{\partial V}\right)_{E}=\frac{k_{B} \kappa}{\Omega} N V^{N-1} \int d \mathbf{s}^{N}(E-U)^{\frac{3 N}{2}-1} \\
-\frac{k_{B} \kappa}{\Omega} V^{N} \int d \mathbf{s}^{N}\left(\frac{3 N}{2}-1\right)(E-U)^{\frac{3 N}{2}-2}\left(\frac{\partial U}{\partial V}\right)_{E} .
\end{array}
$$

Note that the first term on the right-hand side is simply $\frac{N k_{B}}{V}$, using the expression for $\Omega$ from Eq. (27). To simplify the second term on the right, we recognize the average over the phase space and that $K=E-U$, to obtain, for large $N$ :

$$
\left(\frac{\partial S}{\partial V}\right)_{E}=\frac{N k_{B}}{V}-\frac{3 N k_{B}}{2}\left\langle\frac{1}{K}\left(\frac{\partial U}{\partial V}\right)\right\rangle
$$

\section{Obtaining averages from the canonical ensemble}

We now focus on obtaining the averages $\left\langle\frac{1}{K}\right\rangle$ and $\left\langle\frac{1}{K}\left(\frac{\partial U}{\partial V}\right)\right\rangle$, which appear in Eqs. 39p and (43), from the canonical ensemble, to introduce the notion of temperature. Note that, in the thermodynamic limit, all ensembles give similar results (see, e.g., refs. ${ }^{[31}$ and ${ }^{32}$ ). Moreover, our use of the canonical ensemble to obtain the average quantities mentioned above is consistent with Gao et al.'s recent assertion that the Boltzmann distribution is the only one for which thermodynamic entropy equals statistical-mechanical entropy ${ }^{33}$ We consider the same system as in the above section, i.e., $N$ particles of equal mass $m$. We seek to calculate the mean value of $\frac{1}{K}$ in the canonical ensemble. Accordingly,

$$
\left\langle\frac{1}{K}\right\rangle=\frac{\int \exp (-\beta E) \frac{1}{K} d \mathbf{r}^{N} d \mathbf{p}^{N}}{\int \exp (-\beta E) d \mathbf{r}^{N} d \mathbf{p}^{N}}
$$


Since $E=K+U$, we can write:

$$
\left\langle\frac{1}{K}\right\rangle=\frac{\int \exp (-\beta(K+U)) \frac{1}{K} d \mathbf{r}^{N} d \mathbf{p}^{N}}{\int \exp (-\beta(K+U)) d \mathbf{r}^{N} d \mathbf{p}^{N}}
$$

which, by canceling out the $U$ term (because $U$ only depends on $\mathbf{r}^{N}$ ) and using $K=\sum_{i=1}^{N} \frac{\mathbf{p}_{i}^{2}}{2 m}$, simplifies to:

$$
\left\langle\frac{1}{K}\right\rangle=\frac{\int \exp \left(-\beta \frac{\sum_{i=1}^{N} \mathbf{p}_{i}^{2}}{2 m}\right) \frac{2 m}{\sum_{i=1}^{N} \mathbf{p}_{i}^{2}} d \mathbf{p}^{N}}{\int \exp \left(-\beta \frac{\sum_{i=1}^{N} \mathbf{p}_{i}^{2}}{2 m}\right) d \mathbf{p}^{N}} .
$$

Defining $p^{2}=\sum_{i=1}^{N} \mathbf{p}_{i}^{2}$ and recognizing that $d \mathbf{p}^{N}=\frac{2 \pi^{\frac{3 N}{2}}}{\Gamma\left(\frac{3 N}{2}\right)} p^{3 N-1} d p$, based on the surface area of a $3 N$ dimensional hypersphere (see Figure 3), we have:

$$
\left\langle\frac{1}{K}\right\rangle=\frac{\int \exp \left(-\beta \frac{p^{2}}{2 m}\right) \frac{2 m}{p^{2}} \frac{\frac{2 \pi}{\frac{3 N}{2}}}{\Gamma\left(\frac{3 N}{2}\right)} p^{3 N-1} d p}{\int \exp \left(-\beta \frac{p^{2}}{2 m}\right) \frac{2 \pi^{\frac{3 N}{2}}}{\Gamma\left(\frac{3 N}{2}\right)} p^{3 N-1} d p} .
$$

Using the standard Gaussian integral $\int_{0}^{\infty} x^{n} \exp \left(-a x^{2}\right) d x=\frac{\Gamma\left(\frac{n+1}{2}\right)}{2 a^{\frac{n+1}{2}}}$, where $\Gamma$ denotes the Gamma function in mathematics, this can be evaluated to:

$$
\left\langle\frac{1}{K}\right\rangle=\frac{\beta}{\frac{3 N}{2}-1}
$$

For large $N$, one can write the above expression as:

$$
\left\langle\frac{1}{K}\right\rangle=\frac{2 \beta}{3 N}=\frac{2}{3 N k_{B} T}
$$

so that:

$$
\left(\frac{\partial E}{\partial S}\right)_{V}=\frac{1}{k_{B} \beta}
$$

The above derivation showing $\left\langle\frac{1}{K}\right\rangle=\frac{2}{3 N k_{B} T}$ is surprising because it is well known that $\langle K\rangle=\frac{3 N k_{B} T}{2}$, but one would not expect typically that the average of the reciprocal of a quantity would be equal to the reciprocal of the average of that quantity, i.e., $\left\langle\frac{1}{K}\right\rangle=\frac{1}{\langle K\rangle}$. 
In this regard, it may be useful for students to recall that the harmonic mean of a collection of numbers does not, in general, equal the arithmetic mean of the same numbers. Further, it is gratifying that although we have assumed that $N$ is large, the same factor of $\left(\frac{3 N}{2}-1\right)$ appears in both Eqs. 38 and 48 , which implies they will cancel while calculating $\left(\frac{\partial E}{\partial S}\right)_{V}$.

We now move to calculate the mean value of $\frac{1}{K}\left(\frac{\partial U}{\partial V}\right)$ in the canonical ensemble:

$$
\left\langle\frac{1}{K}\left(\frac{\partial U}{\partial V}\right)\right\rangle=\frac{\int \exp (-\beta E) \frac{1}{K}\left(\frac{\partial U}{\partial V}\right) d \mathbf{r}^{N} d \mathbf{p}^{N}}{\int \exp (-\beta E) d \mathbf{r}^{N} d \mathbf{p}^{N}} .
$$

Using $E=K+U$, we can write:

$$
\left\langle\frac{1}{K}\left(\frac{\partial U}{\partial V}\right)\right\rangle=\frac{\int \exp (-\beta K) \frac{1}{K} d \mathbf{p}^{N}}{\int \exp (-\beta K) d \mathbf{p}^{N}} \frac{\int \exp (-\beta U)\left(\frac{\partial U}{\partial V}\right) d \mathbf{r}^{N}}{\int \exp (-\beta U) d \mathbf{r}^{N}},
$$

or,

$$
\left\langle\frac{1}{K}\left(\frac{\partial U}{\partial V}\right)\right\rangle=\left\langle\frac{1}{K}\right\rangle\left\langle\frac{\partial U}{\partial V}\right\rangle
$$

We have already shown above that $\left\langle\frac{1}{K}\right\rangle=\frac{2 \beta}{3 N}$. We now focus our attention on $\left\langle\frac{\partial U}{\partial V}\right\rangle$ and write the internal energy as a sum of two-body $\left(u^{(2)}\right)$, three-body $\left(u^{(3)}\right)$, and higher contributions:

$$
\left\langle\frac{\partial U}{\partial V}\right\rangle=\left\langle\frac{\partial}{\partial V}\left(\sum_{i<j} u^{(2)}\left(\mathbf{r}_{i}, \mathbf{r}_{j}\right)+\sum_{i<j<k} u^{(3)}\left(\mathbf{r}_{i}, \mathbf{r}_{j}, \mathbf{r}_{k}\right)+\ldots\right)\right\rangle
$$

Note that derivations in the literature usually assume pairwise additive two-body interactions, $\frac{3435}{35}$ and do not evaluate $\left\langle\frac{\partial U}{\partial V}\right\rangle$ in the general manner as shown here, including higher-order interaction terms. Higher-order terms are particularly important for molecular systems, where atoms may be involved in bonded (two-body), angular (three-body), and dihedral (four-body) interactions with other atoms. In this regard, another derivation that is well-suited for interatomic potentials and periodic boundary conditions used in molecular 


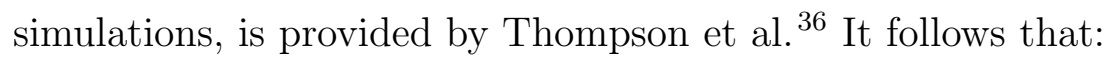

$$
\left\langle\frac{\partial U}{\partial V}\right\rangle=\left\langle\sum_{i<j} \frac{\partial u^{(2)}\left(\mathbf{r}_{i}, \mathbf{r}_{j}\right)}{\partial V}+\sum_{i<j<k} \frac{\partial u^{(3)}\left(\mathbf{r}_{i}, \mathbf{r}_{j}, \mathbf{r}_{k}\right)}{\partial V}+\ldots\right\rangle
$$

which can also be written as:

$$
\left\langle\frac{\partial U}{\partial V}\right\rangle=\left\langle\frac{1}{2 !} \sum_{i \neq j} \frac{\partial u^{(2)}}{\partial V}+\frac{1}{3 !} \sum_{i \neq j \neq k} \frac{\partial u^{(3)}}{\partial V}+\ldots\right\rangle
$$

Thus,

$$
\begin{array}{r}
\left\langle\frac{\partial U}{\partial V}\right\rangle=\frac{1}{2}\left\langle\sum_{i \neq j} \frac{\partial u^{(2)}}{\partial \mathbf{r}_{i}} \cdot \frac{\partial \mathbf{r}_{i}}{\partial V}+\frac{\partial u^{(2)}}{\partial \mathbf{r}_{j}} \cdot \frac{\partial \mathbf{r}_{j}}{\partial V}\right\rangle \\
+\frac{1}{6}\left\langle\sum_{i \neq j \neq k} \frac{\partial u^{(3)}}{\partial \mathbf{r}_{i}} \cdot \frac{\partial \mathbf{r}_{i}}{\partial V}+\frac{\partial u^{(3)}}{\partial \mathbf{r}_{j}} \cdot \frac{\partial \mathbf{r}_{j}}{\partial V}+\frac{\partial u^{(3)}}{\partial \mathbf{r}_{k}} \cdot \frac{\partial \mathbf{r}_{k}}{\partial V}\right\rangle \\
+\langle\ldots\rangle .
\end{array}
$$

Recognizing that $\frac{\partial u^{(m)}}{\partial \mathbf{r}_{i}}=-\mathbf{F}_{i}^{(m)}$, we can write:

$$
\begin{array}{r}
\left\langle\frac{\partial U}{\partial V}\right\rangle=-\frac{1}{2}\left\langle\sum_{i \neq j} \mathbf{F}_{i}^{(2)} \cdot \frac{\partial \mathbf{r}_{i}}{\partial V}+\mathbf{F}_{j}^{(2)} \cdot \frac{\partial \mathbf{r}_{j}}{\partial V}\right\rangle \\
-\frac{1}{6}\left\langle\sum_{i \neq j \neq k} \mathbf{F}_{i}^{(3)} \cdot \frac{\partial \mathbf{r}_{i}}{\partial V}+\mathbf{F}_{j}^{(3)} \cdot \frac{\partial \mathbf{r}_{j}}{\partial V}+\mathbf{F}_{k}^{(3)} \cdot \frac{\partial \mathbf{r}_{k}}{\partial V}\right\rangle \\
+\langle\ldots\rangle .
\end{array}
$$

Now, two simplifications can be made. First, the sums inside the angular brackets are identical and cancel out the respective fractional term $(1 / 2,1 / 6, \ldots)$. Second, using scaled coordinates, one can show that $\frac{\partial \mathbf{r}_{i}}{\partial V}=\frac{\mathbf{r}_{i}}{3 V}$. It follows that:

$$
\left\langle\frac{\partial U}{\partial V}\right\rangle=-\frac{1}{3 V}\left\langle\sum_{i} \mathbf{F}_{i}^{(2)} \cdot \mathbf{r}_{i}\right\rangle-\frac{1}{3 V}\left\langle\sum_{i} \mathbf{F}_{i}^{(3)} \cdot \mathbf{r}_{i}\right\rangle+\langle\ldots\rangle
$$


or,

$$
\left\langle\frac{\partial U}{\partial V}\right\rangle=-\frac{1}{3 V}\left\langle\sum_{i}\left(\mathbf{F}_{i}^{(2)}+\mathbf{F}_{i}^{(3)}+\ldots\right) \cdot \mathbf{r}_{i}\right\rangle
$$

Since $\left(\mathbf{F}_{i}^{(2)}+\mathbf{F}_{i}^{(3)}+\ldots\right)$ equals the total force acting on atom $i$ due to all other particles, we have:

$$
\left\langle\frac{\partial U}{\partial V}\right\rangle=-\frac{1}{3 V}\left\langle\sum_{i} \mathbf{F}_{i} \cdot \mathbf{r}_{i}\right\rangle
$$

Putting the two averages together, we obtain:

$$
\left\langle\frac{1}{K}\left(\frac{\partial U}{\partial V}\right)\right\rangle=-\frac{2 \beta}{9 N V}\left\langle\sum_{i} \mathbf{F}_{i} \cdot \mathbf{r}_{i}\right\rangle .
$$

Using Eq. (62) in Eq. (43), we obtain

$$
\left(\frac{\partial S}{\partial V}\right)_{E}=\frac{N k_{B}}{V}+\frac{\beta k_{B}}{3 V}\left\langle\sum_{i} \mathbf{F}_{i} \cdot \mathbf{r}_{i}\right\rangle
$$

\section{The virial theorem}

One can link $\left(\frac{\partial S}{\partial V}\right)_{E}$ obtained above to the system pressure via Clausius's virial theorem. .273738 Physically, this amounts to equating the "thermodynamic" and "mechanical" pressures in the system, with the former arising from statistical mechanics and the latter arising from the virial theorem. We adapt the derivation for the virial theorem presented by Landau and Lifshitz. ${ }^{39}$ Consider Clausius's virial function defined as:

$$
W\left(\mathbf{r}^{N}, \mathbf{F}^{N}\right)=\sum_{i=1}^{N} \mathbf{r}_{i} \cdot \mathbf{F}_{i}^{\text {tot }}
$$

where $N$ denotes the number of particles in the system, $\mathbf{r}$ the position vector of particle $i$, and $\mathbf{F}_{i}^{\text {tot }}$ the total force (internal plus external) acting on particle $i$. If we average the virial 
over time, we get:

$$
\langle W\rangle=\lim _{t \rightarrow \infty} \frac{1}{t} \int_{0}^{t} d \tau \sum_{i=1}^{N} \mathbf{r}_{i} \cdot \mathbf{F}_{i}^{t o t} .
$$

Using $\mathbf{F}_{i}^{t o t}=m_{i} \ddot{\mathbf{r}}_{i}$, we get:

$$
\langle W\rangle=\lim _{t \rightarrow \infty} \frac{1}{t} \int_{0}^{t} d \tau \sum_{i=1}^{N} \mathbf{r}_{i} \cdot m_{i} \ddot{\mathbf{r}}_{i}
$$

Now, note that at long times, the temporal average of the derivative of a bounded function is zero. For example, the average velocity of a particle moving around in the system is zero over a long period of time, because the displacement of the particle is very small, whereas the averaging time period can be made infinitely large. Formally, this can be written as:

$$
\left\langle\frac{d \mathbf{r}_{i}}{d t}\right\rangle=\lim _{t \rightarrow \infty} \frac{1}{t} \int_{0}^{t} \frac{d \mathbf{r}_{i}(\tau)}{d \tau} d \tau=\lim _{t \rightarrow \infty} \frac{\mathbf{r}_{i}(t)-\mathbf{r}_{i}(0)}{t}=0
$$

Since the position and velocity of each particle are bounded, it follows that:

$$
\left\langle\frac{d\left(\mathbf{r}_{i} \cdot \dot{\mathbf{r}}_{i}\right)}{d t}\right\rangle=0
$$

or,

$$
\left\langle\mathbf{r}_{i} \cdot \ddot{\mathbf{r}}_{i}+\left|\dot{\mathbf{r}}_{i}\right|^{2}\right\rangle=0
$$

Hence,

$$
\left\langle\mathbf{r}_{i} \cdot \ddot{\mathbf{r}}_{i}\right\rangle=-\left\langle\left|\dot{\mathbf{r}}_{i}\right|^{2}\right\rangle
$$

so that,

$$
\langle W\rangle=\left\langle\sum_{i=1}^{N} \mathbf{r}_{i} \cdot m_{i} \ddot{\mathbf{r}}_{i}\right\rangle=-\left\langle\sum_{i=1}^{N} m_{i}\left|\dot{\mathbf{r}}_{i}\right|^{2}\right\rangle .
$$

Now, $m_{i}\left|\dot{\mathbf{r}}_{i}\right|^{2}=2 K_{i}$, where $K_{i}$ denotes the kinetic energy of particle $i$, so that:

$$
\langle W\rangle=\left\langle\sum_{i=1}^{N} \mathbf{r}_{i} \cdot \mathbf{F}_{i}^{t o t}\right\rangle=-2 N\left\langle K_{i}\right\rangle
$$


Using the equipartition theorem of statistical mechanics, $\frac{40}{40}\left\langle K_{i}\right\rangle=\frac{3}{2 \beta}$ and thus:

$$
\langle W\rangle=\left\langle\sum_{i=1}^{N} \mathbf{r}_{i} \cdot \mathbf{F}_{i}^{t o t}\right\rangle=-\frac{3 N}{\beta} .
$$

Now, $\mathbf{F}_{i}^{t o t}=\mathbf{F}_{i}^{e x t}+\mathbf{F}_{i}^{i n t}$, where $\mathbf{F}_{i}^{\text {ext }}$ arises due to the forces exerted by the walls of the system with volume $V$. Analogously, one can write: $W^{t o t}=W^{e x t}+W^{i n t}$. If $\mathbf{r}$ denotes the vector connecting the origin to a boundary element $d A$, one can write:

$$
W^{e x t}=\sum_{i=1}^{N} \mathbf{r}_{i} \cdot \mathbf{F}_{i}^{e x t}=\int \mathbf{r} \cdot(-P d A) \mathbf{n}
$$

where $\mathbf{n}$ denotes the unit vector normal to the wall and pointing outside the system boundary. Using the divergence theorem, we obtain:

$$
W^{e x t}=-P \int \operatorname{div}(\mathbf{r}) d V=-3 P V
$$

One could also have shown the above equality for the cuboidal system that we have considered as $W^{\text {ext }}=L_{x}\left(-P L_{y} L_{z}\right)+L_{y}\left(-P L_{x} L_{z}\right)+L_{z}\left(-P L_{x} L_{y}\right)=-3 P V$, where $V=L_{x} L_{y} L_{z}$. It follows that:

$$
\langle W\rangle=\left\langle\sum_{i=1}^{N} \mathbf{r}_{i} \cdot \mathbf{F}_{i}^{i n t}\right\rangle-3 P V=-\frac{3 N}{\beta},
$$

or,

$$
P=\frac{N}{\beta V}+\frac{1}{3 V}\left\langle\sum_{i=1}^{N} \mathbf{r}_{i} \cdot \mathbf{F}_{i}^{i n t}\right\rangle .
$$

\section{Putting it all together}

At this point, we can go back to the total differential for the internal energy as specified by Eqs. $31-(33)$ :

$$
d E=\frac{d S}{\left(\frac{\partial S}{\partial E}\right)_{V}}-\frac{d V\left(\frac{\partial S}{\partial V}\right)_{E}}{\left(\frac{\partial S}{\partial E}\right)_{V}} .
$$


Using $\left(\frac{\partial S}{\partial E}\right)_{V}$ from Eq. 50 and $\left(\frac{\partial S}{\partial V}\right)_{E}$ from Eq. 63, and recognizing that $\beta=\frac{1}{k_{B} T}$, as mentioned in Section IV, we have:

$$
d E=T d S-\left(\frac{N k_{B} T}{V}+\frac{1}{3 V}\left\langle\sum_{i} \mathbf{F}_{i} \cdot \mathbf{r}_{i}\right\rangle\right) d V
$$

Further, in Eq. (77), which is a statement of Clausius' virial theorem, we have showed that $\left(\frac{N k_{B} T}{V}+\frac{1}{3 V}\left\langle\sum_{i} \mathbf{F}_{i} \cdot \mathbf{r}_{i}\right\rangle\right)$ is equal to the pressure, $P$, of the system. It follows that $d E=T d S-P d V$ is the equation that relates small changes in the system internal energy, its entropy, and its volume, via the temperature and pressure. It may be appealing to students that this equation falls out of a systematic mathematical analysis based on classical and statistical mechanics.

\section{Conclusions}

In this work, we have used the principles of classical statistical mechanics to obtain the combined first and second law of thermodynamics. Specifically, we used the microcanonical $(N V E)$ ensemble to write down the partition function for a monoatomic system of matter and used differential calculus to establish the result. Some new aspects of this derivation include: (i) showing the average of the inverse kinetic energy of the system to be equal to the inverse of the average kinetic energy; (ii) providing physical interpretation of the entropy expression in the microcanionical ensemble in terms of interparticle interactions/external forces; (iii) establishing an expression for the system pressure including many-body interactions, and (iv) introducing pressure in the statistical-mechanical description via Clausius's virial theorem. We hope that learners and educators benefit from the self-contained nature of this exposition linking thermodynamics and statistical mechanics. This proof may also be extended to molecular systems and to systems of particles with unequal masses, as desired. Such exercises could be assigned to the students to improve their understanding of the subject. Students could also be encouraged to re-work parts of the derivation presented herein by themselves. 
To conclude, our work provides a new way of teaching students the use of the microcanonical formulation of statistical mechanics to derive fundamental thermodynamic relationships.

\section{Acknowledgement}

A.G.R. thanks Prof. Ganapathy Ayappa, Prof. Prabal Maiti, Prof. Sudeep Punnathanam, Dr. Dimitrios Fraggedakis, and Dr. Robert T. Hanlon for their valuable feedback on the manuscript.

\section{References}

(1) Puvvada, S.; Blankschtein, D. Molecular-Thermodynamic Approach to Predict Micellization, Phase Behavior and Phase Separation of Micellar Solutions. I. Application to Nonionic Surfactants. The Journal of Chemical Physics 1990, 92, 3710.

(2) Gupta, A.; Govind Rajan, A.; Carter, E. A.; Stone, H. A. Thermodynamics of Electrical Double Layers with Electrostatic Correlations. The Journal of Physical Chemistry C 2020, 124, 26830-26842.

(3) Zhang, W.; Smith, J. R.; Wang, X.-G. Thermodynamics from Ab Initio Computations. Physical Review B 2004, 70, 024103.

(4) Bazant, M. Z. Theory of Chemical Kinetics and Charge Transfer based on Nonequilibrium Thermodynamics. Accounts of Chemical Research 2013, 46, 1144-1160.

(5) Cheng, B.; Engel, E. A.; Behler, J.; Dellago, C.; Ceriotti, M. Ab Initio Thermodynamics of Liquid and Solid Water. Proceedings of the National Academy of Sciences 2019, 116, $1110-1115$.

(6) Haynie, D. T. Biological Thermodynamics; Cambridge University Press, 2001. 
(7) Chakraborty, A. K.; Košmrlj, A. Statistical Mechanical Concepts in Immunology. Annual Review of Physical Chemistry 2010, 61, 283-303.

(8) Frenkel, D.; Smit, B. Understanding Molecular Simulation: From Algorithms to Applications; Elsevier Inc., 2002.

(9) Allen, M. P.; Tildesley, D. J. Computer Simulation of Liquids; Oxford University Press, 2017.

(10) Jaynes, E. T. Information Theory and Statistical Mechanics. Physical Review 1957, 106, 620-630.

(11) Park, J.; Newman, M. E. J. Statistical Mechanics of Networks. Physical Review E 2004, 70, 066117.

(12) Hanlon, R. T. Block by Block: The Historical and Theoretical Foundations of Thermodynamics; Oxford University Press, 2020.

(13) Gibbs, J. W. Elementary Principles in Statistical Mechanics; Courier Corporation, 2014.

(14) Salem, W. K. A.; Fröhlich, J. Status of the Fundamental Laws of Thermodynamics. Journal of Statistical Physics 2007, 126, 1045-1068.

(15) Lieb, E. H.; Yngvason, J. The Physics and Mathematics of the Second Law of Thermodynamics. Physics Reports 1999, 310, 1-96.

(16) Gemmer, J.; Otte, A.; Mahler, G. Quantum Approach to a Derivation of the Second Law of Thermodynamics. Physical Review Letters 2001, 86, 1927-1930.

(17) Tasaki, H. Quantum Statistical Mechanical Derivation of the Second Law of Thermodynamics: A Hybrid Setting Approach. Physical Review Letters 2016, 116, 170402. 
(18) Masanes, L.; Oppenheim, J. A General Derivation and Quantification of the Third Law of Thermodynamics. Nature Communications 2017, 8, 14538.

(19) Strasberg, P.; Winter, A. First and Second Law of Quantum Thermodynamics: A Consistent Derivation Based on a Microscopic Definition of Entropy. PRX Quantum 2021, 2, 030202.

(20) Swaney, R. E.; Bird, R. B. The First and Second Laws of Thermodynamics. Physics of Fluids 2019, 31, 097105.

(21) McQuarrie, D. A. Statistical Mechanics; Harper \& Row Publishers, 1976.

(22) Hill, T. L. An Introduction to Statistical Thermodynamics; Dover Publications Inc., 1986.

(23) Kardar, M. Statistical Physics of Particles; Cambridge University Press, 2007.

(24) Swendsen, R. Thermodynamics, Statistical Mechanics and Entropy. Entropy 2017, 19, 603.

(25) Swendsen, R. H.; Wang, J.-S. Gibbs Volume Entropy is Incorrect. Physical Review E 2015, 92, 020103.

(26) Frenkel, D.; Warren, P. B. Gibbs, Boltzmann, and Negative Temperatures. American Journal of Physics 2015, 83, 163-170.

(27) Pathria, R. K.; Beale, P. D. Statistical Mechanics, 3rd ed.; Elsevier, 2011.

(28) Reif, F. Fundamentals of Statistical and Thermal Physics; Waveland Press, Inc., 2009.

(29) Tolman, R. C. The Principles of Statistical Mechanics; Oxford at the Clarendon Press, 1938.

(30) Swendsen, R. H. An Introduction to Statistical Mechanics and Thermodynamics; Oxford University Press, 2012. 
(31) Dobrushin, R. L.; Tirozzi, B. The Central Limit Theorem and The Problem of Equivalence of Ensembles. Communications in Mathematical Physics 1977, 54, 173-192.

(32) Georgii, H.-O. The Equivalence of Ensembles for Classical Systems of Particles. Journal of Statistical Physics 1995, 80, 1341-1378.

(33) Gao, X.; Gallicchio, E.; Roitberg, A. E. The Generalized Boltzmann Distribution is the Only Distribution in Which the Gibbs-Shannon Entropy Equals the Thermodynamic Entropy. The Journal of Chemical Physics 2019, 151, 034113.

(34) Tsai, D. H. The Virial Theorem and Stress Calculation in Molecular Dynamics. The Journal of Chemical Physics 1979, 70, 1375-1382.

(35) Morante, S.; Rossi, G. C.; Testa, M. The Stress Tensor of a Molecular System: An Exercise in Statistical Mechanics. The Journal of Chemical Physics 2006, 125, 034101.

(36) Thompson, A. P.; Plimpton, S. J.; Mattson, W. General Formulation of Pressure and Stress Tensor for Arbitrary Many-Body Interaction Potentials under Periodic Boundary Conditions. The Journal of Chemical Physics 2009, 131, 154107.

(37) Clausius, R. XVI. On A Mechanical Theorem Applicable to Heat. The London, Edinburgh, and Dublin Philosophical Magazine and Journal of Science 1870, 40, 122-127.

(38) Marc, G.; McMillan, W. G. In Advances In Chemical Physics; Prigogine, I., Rice, S. A., Eds.; John Wiley \& Sons, Inc., 1985; pp 209-361.

(39) Landau, L. D.; Lifshitz, E. M. Statistical Physics, Part 1: Volume 5; ButterworthHeinemann, 1980.

(40) Huang, K. Statistical Mechanics, 2nd ed.; John Wiley \& Sons, Inc., 1987. 
TOC Graphic

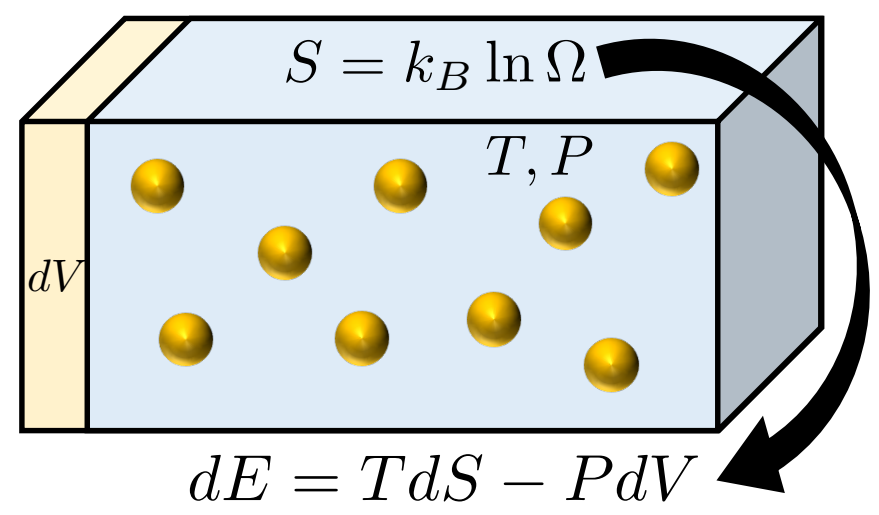

